\title{
Sliding Mode Control of Double Inverted Pendulum Based on a Novel Approach Law
}

\section{L.F.SUN}

School of Automation Engineering, Northeast Dianli University, Jilin, Jilin, China

C.Y.FU

School of Automation Engineering, Northeast Dianli University, Jilin, Jilin, China

\begin{abstract}
In this paper, a sliding mode controller based on new type of approach law is applied to double inverted pendulum(DIP) in order to weaken the chattering and shorten the approaching time, ensure the system to achieve better dynamic quality and stabilize the DIP system around equilibrium states. Further, theoretical proof is given to demonstrate the feasibility of the proposed method. Experimental results of implementing proposed controller show a high performance of it in comparison to traditional exponential approach law.
\end{abstract}

KEYWORD: inverted pendulum; sliding mode; approach law; chattering

\section{INTRODUCTION}

The inverted pendulum system is a inherently underactuated system with high order, nonlinear, multivariate, and strong coupling. Since the feasibility and performance of the algorithms can be tested on it and it's similarity in control of operation of satellite and robot walking with two legs, it has always been the hot topic in control fields.

The sliding mode control(SMC) was proposed by Utkin. It is a robust discontinuous controller which is insensitive to the parameter variation and disturbance with easy implementation and quick response. But the main drawback, chattering, can damage the system performance and even lead to instability since it's high-frequency oscillation. Specialists are committed to solving this problem. Gao proposed a exponential reaching law, eliminating chattering and cutting reaching time down efficiently.[6] The reaching component of SMC is replaced by an IT2FLS to reduce chattering taking into consideration possible system uncertainties.[1] A robust adaptive sliding control law is designed, not having sign function, to eliminate the chattering and to ensure the occurrence of the sliding motion even when systems are perturbed with unknown uncertainties and external disturbances.[2] A high-order sliding mode(HOSM) control is proposed to handle the chattering problem with the chattering-free behavior and robustness against external disturbances.[4] A robust discretetime SMC algorithm coupled with an uncertainty estimator based on sliding modes is used for a planar robotic manipulator. Good tracking performance and robustness are obtained when payload perturbations and inaccuracies disturbances exist. [5]

In this paper, double inverted pendulum model is presented. A novel approach law is applied to SMC controller to weaken chattering and shorten approach time. Simulation is given to stabilize the system and finally we draw some conclusions.

\section{DOUBLE INVERTED PENDULUM SYSTEM FORMULATION}

The DIP system physical model is shown as Fig.1.

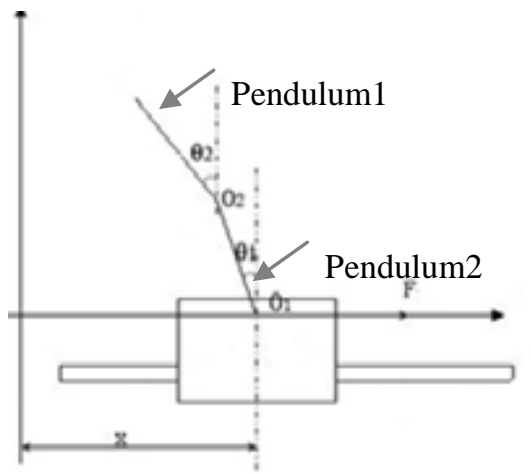

Figure 1. Physical model of DIP system.

Which $\theta_{1}, \theta_{2}, x, \mathbf{M}, m_{1}, m_{2}, m_{3} l_{1}, l_{2}$ represents the angle of pendulum1,2; cart position;mass of cart, pendulum1,2 and pivot; distance from turning center to mass center of the pendulum 1,2 respectively.

Lagrange motion equations is used to obtain the DIP system nonlinear model as follow: 
$\ddot{\theta}_{1}=\frac{\left(\left(\begin{array}{l}-2 g m_{1} \sin \theta_{1}-4 g m_{2} \sin \theta_{1}-4 m_{3} g \sin \theta_{1} \\ +3 m_{2} g \cos \left(\theta_{2}-\theta_{1}\right) \sin \theta_{2}+6 m_{2} l_{1} \cos \left(\theta_{2}-\theta_{1}\right) \sin \left(\theta_{2}-\theta_{1}\right) \dot{\theta}_{1}^{2} \\ +4 m_{2} l_{2} \sin \left(\theta_{1}-\theta_{2}\right) \dot{\theta}_{2}^{2}-2 m_{1} \ddot{x} \cos \theta_{1}-4 m_{2} \ddot{x} \cos \theta_{1} \\ -4 m_{3} \ddot{x} \cos \theta_{1}+3 m_{2} \ddot{x} \cos \left(\theta_{1}-\theta_{2}\right) \cos \theta_{2}\end{array}\right)\right)}{\left(2 l_{1}\left(-4 m_{1}-12 m_{2}-12 m_{3}+9 m_{2} \cos ^{2}\left(\theta_{1}-\theta_{2}\right)\right)\right)}$

$\ddot{\theta}_{2}=\frac{\left(\begin{array}{l}4 \\ -\frac{4}{9} m_{2}\left(m_{1}+3\left(m_{2}+m_{3}\right)\right) l_{1}^{2} l_{2}\left(-3 g \sin \theta_{2}-6 l_{1} \dot{\theta}_{1}^{2} \sin \left(\theta_{1}-\theta_{2}\right)-3 \ddot{x} \cos \theta_{2}\right) \\ +\frac{2}{3} m_{2} l_{1}^{2} l_{2} \cos \left(\theta_{1}-\theta_{2}\right)\left(6 m_{2} l_{2} \dot{\theta}_{2}^{2} \sin \left(\theta_{1}-\theta_{2}\right)-3\left(m_{1}+2\left(m_{2}+m_{3}\right)\right)\left(g \sin \theta_{1}+\ddot{x} \cos \theta_{1}\right)\right)\end{array}\right)}{\left.-\frac{16}{9} m_{2}\left(m_{1}+3\left(m_{2}+m_{3}\right)\right)\right)_{1}^{2} l_{2}^{2}+4 m_{2}^{2} l_{1}^{2} l_{2}^{2} \cos (\theta 1-\theta 2)}$

$\Gamma=\left[x, \theta_{1}, \theta_{2}, \dot{x}, \dot{\theta}_{1}, \dot{\theta}_{2}\right]$ is selected as state variables.After linearization around equilibrium points, the linear model is as follows:

$\left\{\begin{array}{l}\dot{\Gamma}=\mathrm{G} \Gamma+\mathrm{Hu} \\ y=C \Gamma\end{array}\right.$

Where $\mathrm{u}$ is control force applying onto the cart, $\mathrm{G}, \mathrm{H}, \mathrm{C}$ are as follow:

$\mathrm{G}=\left[\begin{array}{cccccc}0 & 0 & 0 & 1 & 0 & 0 \\ 0 & 0 & 0 & 0 & 1 & 0 \\ 0 & 0 & 0 & 0 & 0 & 1 \\ 0 & 0 & 0 & 0 & 0 & 0 \\ 0 & 86.69 & -21.62 & 0 & 0 & 0 \\ 0 & -40.31 & 39.45 & 0 & 0 & 0\end{array}\right]$

$H=\left[\begin{array}{c}0 \\ 0 \\ 0 \\ 1 \\ 6.64 \\ -0.088\end{array}\right] C=\left[\begin{array}{llllll}1 & 0 & 0 & 0 & 0 & 0 \\ 0 & 1 & 0 & 0 & 0 & 0 \\ 0 & 0 & 1 & 0 & 0 & 0\end{array}\right]$

\section{SLIDING MODE CONTROL}

Sliding Mode Control is a kind of discontinuous control,which can be expressed as:

$u(x)=\left\{\begin{array}{l}u^{+}(x), s(x)<0 \\ u^{-}(x), s(x)>0\end{array}\right.$

Switch surface $s(x)$ must be reached in finite time.The control law guarantee system slides to the equilibrium points along the surface instead of passing through it back and forth,namely reducing chattering.Theoretical proof must be given to ensure accessibility of sliding mode.

\subsection{Switching surface design}

For DIP linear state-space model $\dot{\Gamma}=\mathrm{G} \Gamma+\mathrm{Hu}$,the switching face can be defined as a linear combination of state variables:

$$
\Psi(\Gamma)=\mathrm{K} \Gamma=k_{1} x+k_{2} \theta_{1}+k_{3} \theta_{2}+k_{4} \dot{x}+k_{5} \dot{\theta}_{1}+k_{6} \dot{\theta}_{2}
$$

The $\mathrm{K}$ is given by Ackermann formulation [3]:
$K=e^{T} P(G)$

$e^{T}=\left[\begin{array}{llllll}0 & 0 & 0 & 0 & 0 & 1\end{array}\right]\left[\begin{array}{llllll}H & G H & G^{2} H & G^{3} H & G^{4} H & G^{5} H\end{array}\right]^{-1}$

$P(w)=\left(w-w_{1}\right)\left(w-w_{2}\right)\left(w-w_{3}\right)\left(w-w_{4}\right)\left(w-w_{5}\right)$

Which $w_{i}$ is the target pole.

\subsection{Control law design}

Traditional exponential approach law ensures a large speed to approach switching face, while the final speed is a positive constant instead of zero, never letting the system remove chattering theoretical. Traditional power approach law provides a gentle speed when closer to the surface to eliminate chattering, undesirable extending reaching time still damages the dynamic property.

In order to balance the advantages and disadvantages of the two reaching laws, to get better control performance, in this section, a new type of approach law is designed as follow:

$$
\dot{\Psi}=-\rho_{1}|\psi|^{\lambda_{1}} \operatorname{sgn}(\psi)-\rho_{2}|\psi|^{\lambda_{2}} \operatorname{sgn}(\Psi)
$$

Where $\rho_{1}>0, \rho_{2}>0, \lambda_{1}>1,1>\lambda_{2}>0$.

When the system away from the sliding surface $(|\psi|>1)$, the first term plays a leading role, otherwise $(|\psi|<1)$ the second term does. In this way, the proposed law preserves the advantages of power approach law, which sliding into the surface gently with the purpose of decreasing chattering and enhancing approach speed in reaching stage to be aimed at getting better control performance simultaneously.

On the basis of the linear mode of DIP and approach law, the control law can be shown as follow:

$$
u=-(K H)^{-1}\left(K G \Gamma+\rho_{1}|\Psi|^{\lambda_{1}} \operatorname{sgn}(\Psi)+\rho_{2}|\Psi|^{\lambda_{2}} \operatorname{sgn}(\Psi)\right)
$$

\subsection{Stability analysis}

To ensure the stability of control system, we choose a Lyapunov function as:

$$
\begin{aligned}
& V=\frac{1}{2} \Psi^{2} \\
& \dot{V}=\Psi \dot{\Psi}=-\rho_{1}|\Psi|^{\lambda_{1}+1}-\rho_{2}|\Psi|^{\lambda_{2}+1}<0
\end{aligned}
$$

It shows surface $\Psi(\Gamma)$ can be reached in finite time from any $\Psi(0)$, meeting the requirements of the sliding mode accessibility.

$\Psi(0)>1$ is taken as an example to calculate reaching time. The process is divided into two parts.

Stage one: $\Psi(0)$ moving to $\Psi(\Gamma)=1$

Due to $\Psi(0)>1, \lambda_{1}>1,1>\lambda_{2}>0$, the first term in formula(9) plays a main role, while ignoring the other term, the approach law can be written as: 
$\dot{\Psi}=-\rho_{1}|\psi|^{\lambda_{1}} \operatorname{sgn}(\psi)$

Compute integral of formula(13) on both sides:

$\Psi^{1-\lambda_{1}}=-\left(1-\lambda_{1}\right) \rho_{1} t_{1}+\Psi(0)^{1-\lambda_{1}}$

Then we get the approach time in stage one as:

$t_{1}=\frac{1-\Psi(0)^{1-\lambda_{1}}}{\rho_{1}\left(\lambda_{1}-1\right)}$

Stage two: $\Psi(\Gamma)=1$ moving to $\Psi(\Gamma)=0$

Because of $\Psi(0)>1, \quad \lambda_{1}>1,1>\lambda_{2}>0$, the second term in formula(9) plays a main role instead, the approach law can be written as:

$\dot{\Psi}=-\rho_{2}|\psi|^{\lambda_{2}} \operatorname{sgn}(\Psi)$

Compute integral of formula(16) on both sides:

$\Psi^{1-\lambda_{2}}=-\left(1-\lambda_{2}\right) \rho_{2} t_{2}+1$

Then we get the approach time in stage two as:

$t_{2}=\frac{1}{\rho_{2}\left(1-\lambda_{2}\right)}$

The whole approach time is obtained as:

$t=t_{1}+t_{2}=\frac{1-\Psi(0)^{1-\lambda_{1}}}{\rho_{1}\left(\lambda_{1}-1\right)}+\frac{1}{\rho_{2}\left(1-\lambda_{2}\right)}$

Analogously, when $\Psi(0)<-1$, the approach time can be written as:

$t=t_{1}+t_{2}=\frac{1+\Psi(0)^{1-\lambda_{1}}}{\rho_{1}\left(\lambda_{1}-1\right)}+\frac{1}{\rho_{2}\left(1-\lambda_{2}\right)}$

In fact,real approach time is less than calculation time by ignoring the secondary factors.

\section{SIMULATION}

Figure. 2 shows the controller design using new type approach law in matlab/simulink environment.

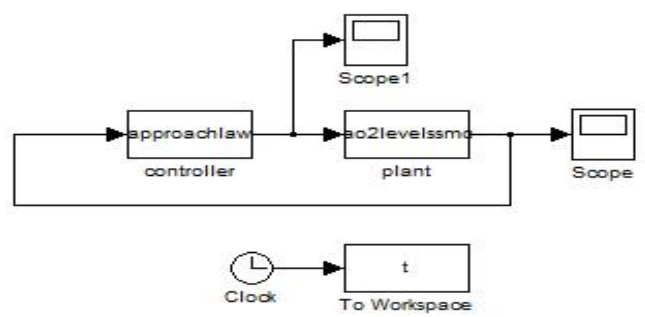

Figure 2. DIP controller design

Seen from repeated experiments, the larger target poles are chosen,the shorter adjusting time the system will get.The overshoot is getting bigger since the faster response speed.Also stronger control force may over operating range of the actuator, damaging control effects. Increasing the value of parameter $\rho_{1}$ and parameter $\lambda_{1}$ can accelerate the approach velocity far away from switch surface. Similarly for $\rho_{2}$ and $\lambda_{2}$, under the premise of smoothly reaching the surface, appropriate value increasing will reduce the reaching time for better approach quality. Considering the above factors, We select the target poles as $-5,-7,-9+4 \mathrm{i}, \quad-9-4 \mathrm{i},-17$, and controller parameters are given like $\rho_{1}=0.2, \rho_{2}=1.5$, $\lambda_{1}=2, \lambda_{2}=0.9$. The initial condition of the states is chosen to be $\Gamma=[0.1,0.1,0.1,0,0,0]$.

The simulation results of proposed controller compared with the traditional exponential approach law are shown in Figure.3-Figure.5:

From figure.3-figure.5 we can see that by employing the proposed method on DIP,pendulum1 and pendulum2 converges to the desired value smoothly in about 1.5 seconds with little overshoot, while the traditional exponential approach law needs longer time to stabilize system balance, with fluctuation and bigger overshoot. Despite the cart needs longer settling time, the system has a low precision of it. It comes to the conclusion easily that the proposed method cuts down the approach time, in the meanwhile, reduces the overshoot to get the system better dynamic quality.

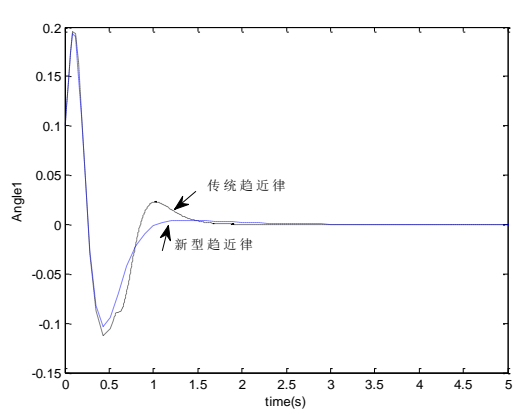

Figure 3. Angle of pendulum1

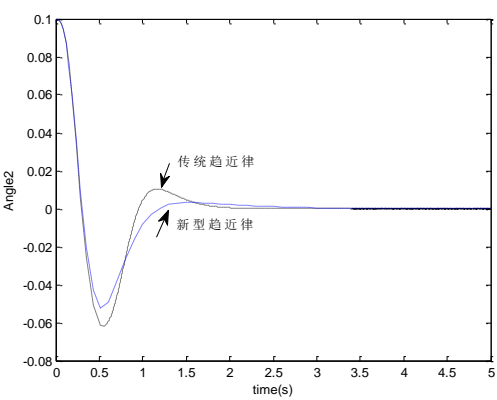

Figure 4. Angle of pendulum2

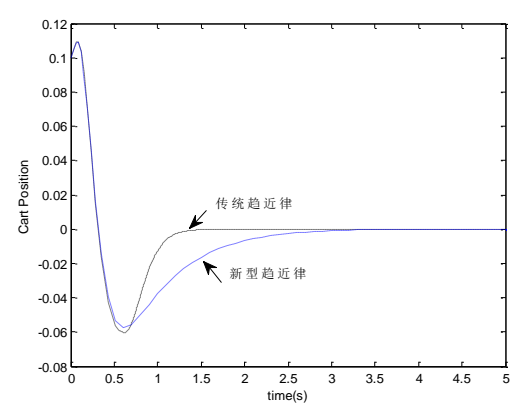

Figure 5. Position of cart 
Figure. 6 and Figure. 7 show the control input of the system:

By comparing,the new type approach law eliminates the chattering obviously.

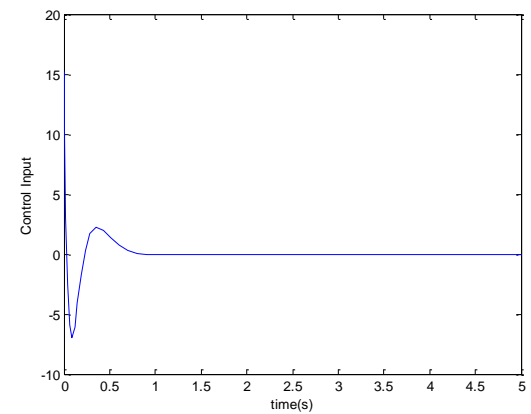

Figure 6. New approach control law

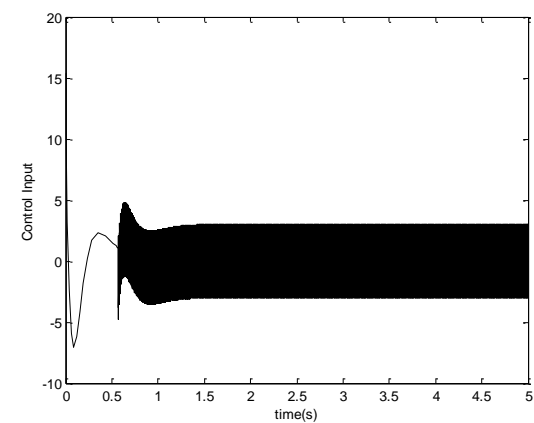

Figure 7. Traditional approach control law

When the disturbance signal $y=0.2 \sin (2 \pi t)$ is added to the system,the simulation results are shown in Figure.8-Figure.10:

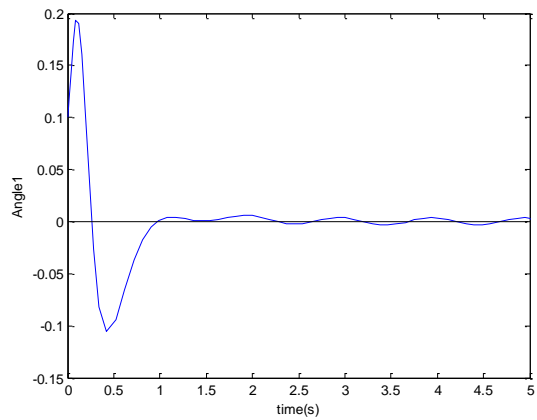

Figure 8. Angle of pendulum1

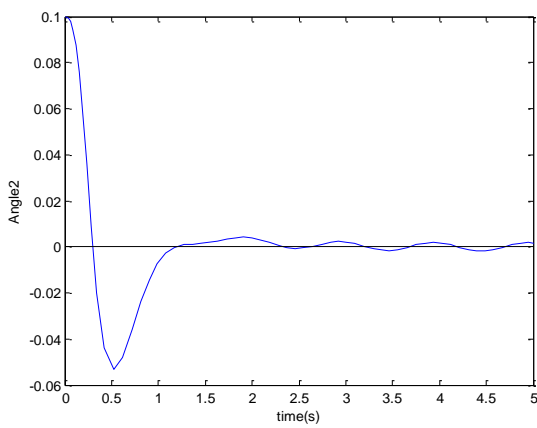

Figure 9. Angle of pendulum2

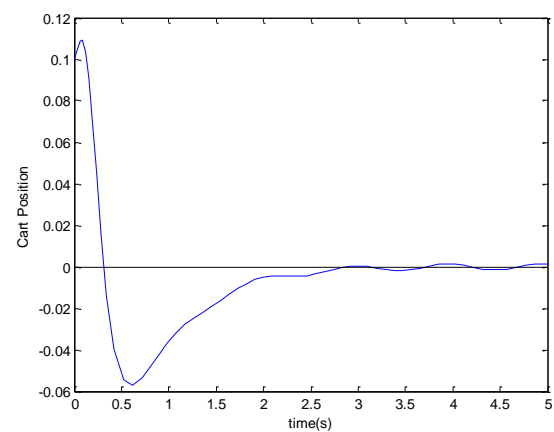

Figure 10. Position of cart

The simulation results demonstrate that the systym outputs can return to equilibrium point rapidly with no more than 0.06 fluctuation range, which well proves that the proposed method has fine anti-interference performance.

\section{CONCLUSION}

A sliding mode controller based on new type of approach law is applied to double inverted pendulum system. The Theoretical proof and compared simulation results all demonstrate that utilizing proposed method can weaken the chattering and shorten the reaching time, meanwhile reduce the overshoot, getting the system better dynamic quality. From the disturbance simulation experiment, we can see the controller is insensitive to external disturbance. A summary of all that, the proposed method is effective with a high control performance.

\section{REFERENCE}

[1] Abdelaal M E \& Emara H M \& Bahgat A. 2013. Interval type 2 fuzzy sliding mode control with application to inverted pendulum on a cart. Industrial Technology (ICIT), 2013IEEE International Conference on. IEEE2013: 100-105.

[2] Aghababa M P \& Akbari M E. A. 2012. Chattering-free robust adaptive sliding mode controller for synchronization of two different chaotic systems with unknown uncertainties and external disturbances. Applied Mathematics and Computation 218(9): 5757-5768.

[3] Ackermann J \& Utkin V. 1998. Sliding mode control design based on Ackermann's formula. Automatic Control, IEEE Transactions on 43(2): 234-237.

[4] Benbouzid M \& Beltran B \& Amirat Y, et al. 2013. HighOrder Sliding Mode Control for DFIG-Based Wind Turbine Fault Ride-Through. Industrial Electronics Society, IECON 2013-39th Annual Conference of the IEEE. IEEE2013: $7670-7674$.

[5] Corradini M L \& Fossi V \& Giantomassi A, et al. 2012. Discrete time sliding mode control of robotic manipulators. Development and experimental validation. Control Engineering Practice2012, 20(8): 816-822.

[6] Gao W B. 1993.On the design of variable structure controller. 